\title{
Multi-scale morphological gradient algorithm based ultra-high-speed directional transmission line protection for internal and external fault discrimination
}

\author{
Elmahdi Khoudry ${ }^{1}$, Abdelaziz Belfqih ${ }^{2}$, Tayeb Ouaderhman ${ }^{3}$, Jamal Boukherouaa ${ }^{4}$, Faissal \\ Elmariami $^{5}$ \\ ${ }^{1,2,4,5}$ Team of Electric Networks and Static Converters. Laboratory of Energy and Electrical Systems., National Higher \\ School of Electricity and Mechanics (ENSEM). Hassan II University, Casablanca, Morocco \\ ${ }^{3}$ MACS Laboratory. FSAC. Hassan 2 University. Casablanca. Morocco
}

\begin{tabular}{l}
\hline \hline Article Info \\
\hline Article history: \\
Received Jan 9, 2019 \\
Revised Apr 5, 2019 \\
Accepted Apr 15, 2019 \\
\hline Keywords: \\
Ultra-high-speed directional \\
protection \\
Internal and external fault \\
discrimination \\
Multi-scale morphological \\
gradient algorithm \\
Traveling waves \\
Fault-induced transients \\
\hline
\end{tabular}

\begin{abstract}
This paper introduces an ultra-high-speed directional transmission line protection scheme based on multi-scale morphological gradient algorithm (MSMGA). The directional protection scheme sets down the rules for determining the fault position in relation to the relaying point. The MSMGA is used to extract the fault-induced transient characteristics contained in the voltage and current signals. The associated signals are formed from these transient characteristics and the polarity of their local modulus maxima allow the discrimination between internal and external faults.
\end{abstract}

Copyright (C) 2019 Insitute of Advanced Engineeering and Science. All rights reserved.

Corresponding Author:

Elmahdi Khoudry,

Laboratory of Energy and Electrical Systems,

National Higher School of Electricity and Mechanics,

Road El Jadida, Km 7, BP: 8118, Oasis-Casablanca, Morocco,

Email: e.khoudry@gmail.com

\section{INTRODUCTION}

Extra High Voltage (EHV) transmission lines constitute an essential part of the electrical power system. Therefore, they should rely on efficient protection schemes capable of removing faults swiftly and selectively to enhance the transient stability and ensuring the continuity of power supply. For these reasons, ultra-high-speed directional protection (UHSDP) schemes have started to be closely studied and broadly implemented in EHV power systems.

Being one of the UHSDP schemes, traveling wave-based directional protection can quickly identify the direction of the fault based on the traveling wave signal [1], [2], [3]. Not only that, but it is also sheltered from the impacts of power swings and current transformer saturation. Nevertheless, it is highly sensitive to the fault inception angle. In fact, it is unable to detect faults under low voltage inception angle because the fault-induced traveling wave is too damped.

Artificial intelligence based directional protection schemes have been put forward as an alternative to the previous method [4], [5], [6], [7], [8]. These schemes are essentially based on artificial neural networks which remain a fast and reliable tool when the directional protection is treated as a pattern recognition prob- 
lem. Nonetheless, neural networks need a large amount of data to perform their supervised learning, which is sometimes not available.

To address the downsides of the traveling wave-based directional protection and of the artificial intelligence techniques, the transient-based protection scheme has been introduced [9], [10]. The implementation of this scheme depends on exploiting the wave equations which links together the transient voltage, the transient current, time and distance. The exploitation of the wave equation consists of extracting the transients (the superimposed quantities) generated by a fault. For this task, two classical methods were proposed. The first one consists of using the Fourier Transform, but this method gives access only to the frequency components of a signal over the period of time in which it exists but does not provide any information about the interval of times at which these frequencies appear. As a result, the Fourier Transform is not suitable to extract the transient signals, which is a non-stationary signal, from the signals measured at different transmission line busbars under the fault condition. The second method is based on the Wavelet Transform [11], [12],[13]. The Wavelet Transform uses a short time window for the analysis of the high-frequency content of a signal and a long time window for low-frequency content. Therefore, the Wavelet Transform provides an accurate time-frequency analysis of the signal. However, the Wavelet Transform algorithm is computationally demanding and sensible to noise.

In this paper, we propose another approach to the ultra-high-speed directional protection based on the multi-scale morphological gradient algorithm. This approach is used for the purpose of discriminating between internal and external faults. It should also be noted, unlike the Fourier Transform and the Wavelet transform, that this method processes the signal in the time domain only. In addition to that, narrow sliding data windows suffice to perform accurate, quick and simple computations. All these advantages make this approach suitable to real-time applications.

\section{MULTI-SCALE MORPHOLOGICAL GRADIENT ALGORITHM (MSMGA)}

Before presenting the MSMGA, we will first start by introducing the different flat morphological functions that intervene in the algorithm [14], [15], [16]. These functions are mathematically defined as follows:

(a) Level $s$ flat positive dilation:

$$
\delta_{s}^{+}[n]= \begin{cases}\max _{1 \leq m \leq l_{s}}\left\{\rho_{s-1}[n+m]\right\} & 1 \leq n \leq l_{x}-l_{s}+1 \\ \rho_{s-1}[n] & l_{x}-l_{s}+2 \leq n \leq l_{x}\end{cases}
$$

(b) Level $s$ flat positive erosion:

$$
\varepsilon_{s}^{+}[n]=\left\{\begin{array}{lr}
\rho_{s-1}[n] & 1 \leq n \leq l_{s}-1 \\
\min _{1 \leq m \leq l_{s}}\left\{\rho_{s-1}[n+m]\right\} & l_{s} \leq n \leq l_{x}
\end{array}\right.
$$

(c) Level $s$ flat negative dilation:

$$
\delta_{s}^{-}[n]=\left\{\begin{array}{lr}
\rho_{s-1}[n] & 1 \leq n \leq l_{s}-1 \\
\max _{1 \leq m \leq l_{s}}\left\{\rho_{s-1}[n+m]\right\} & l_{s} \leq n \leq l_{x}
\end{array}\right.
$$

(d) Level $s$ flat negative erosion:

$$
\varepsilon_{s}^{-}[n]= \begin{cases}\min _{1 \leq m \leq l_{s}}\left\{\rho_{s-1}[n+m]\right\} & 1 \leq n \leq l_{x}-l_{s}+1 \\ \rho_{s-1}[n] & l_{x}-l_{s}+2 \leq n \leq l_{x}\end{cases}
$$

(e) Level $s$ flat positive gradient:

$$
\rho_{s}^{+}[n]=\delta_{s}^{+}[n]-\varepsilon_{s}^{+}[n]
$$

(f) Level $s$ flat negative gradient:

$$
\rho_{s}^{-}[n]=\varepsilon_{s}^{-}[n]-\delta_{s}^{-}[n]
$$


(g) Level $s$ flat gradient:

$$
\rho_{s}[n]=\rho_{s}^{+}[n]+\rho_{s}^{-}[n]
$$

Where

- $x[n]:$ A sampled input signal.

- $\rho_{0}[n]=x[n]$

- $l_{1}$ : The level 1 length of the operators $\max$ and $\min$.

- $l_{s}=2^{s-1} l_{1}$ : The dyadic level $s$ length of the operators $\max$ and $\min$.

- $l_{x}$ : The length of the discrete signal $x[n]$.

To notice the effects of these functions, let's, for instance, consider the signal of Figure 1(a) and restrict our analysis to level 1 flat morphological functions. As depicted in Figure 1(b), the flat positive dilation and the flat positive erosion affect only the ascending edge of the signal. In fact, the ascending edge of the ramped-signal has been expanded to the left by $l_{1}-1$ samples using the function $\delta_{s}^{+}[n]$ (see the signal in red), whereas it has been compressed to the right by $l_{1}-1$ samples using the function $\varepsilon_{s}^{+}[n]$ (see the signal in blue). As illustrated in Figure 1(c), the flat negative dilation and the flat negative erosion affect only the descending edge of the signal. In fact, the descending edge of the ramped-signal has been expanded to the right by $l_{1}-1$ samples using the function $\delta_{s}^{-}[n]$ (see the signal in red), whereas it has been compressed to the left by $l_{1}-1$ samples using the function $\varepsilon_{s}^{-}[n]$ (see the signal in blue).

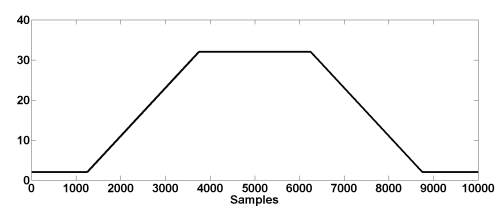

(a)

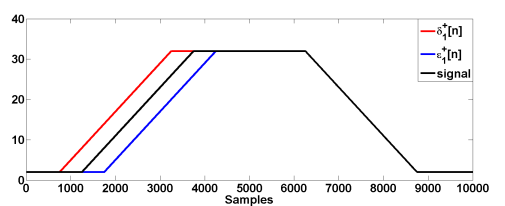

(b)

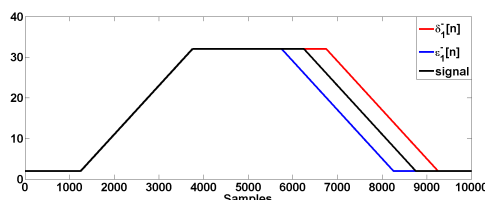

(c)

Figure 1. (a) A ramped-step signal, (b) The ramped-step signal (black), $\delta_{s}^{+}[n]$ (red) and $\varepsilon_{s}^{+}[n]$ (blue), (c) The ramped-step signal (black), $\delta_{s}^{-}[n]$ (red) and $\varepsilon_{s}^{-}[n]$ (blue)

Finally, we deduce from Figures 2(a) and 2(b) that $\rho_{1}^{+}[n]$ and $\rho_{1}^{-}[n]$ not only provide us with information about the moment of waveform changes, but also inform us about the polarities of these changes. The polarity is positive for ascending edges and negative for descending edges. To obtain the whole profile of the waveform changes, it suffices to combine the flat positive gradient and the flat negative gradient in one single flat gradient as shown in Figure 2(c). Hence the definition of level $s$ flat gradient and the flowchart of the MSMGA that is given in Figure 3.

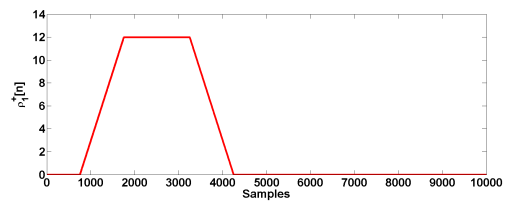

(a)

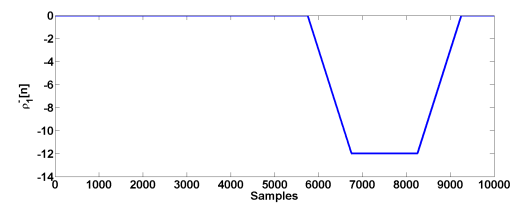

(b)

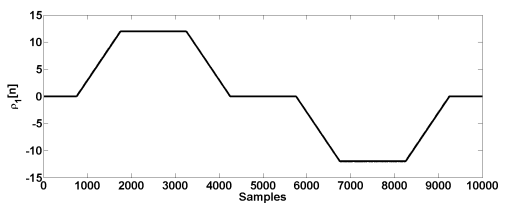

(c)

Figure 2. (a) $\rho_{1}^{+}[n]$ of the ramped-step signal, (b) $\rho_{1}^{-}[n]$ of the ramped-step signal,

(c) $\rho_{1}[n]$ of the ramped-step signal 


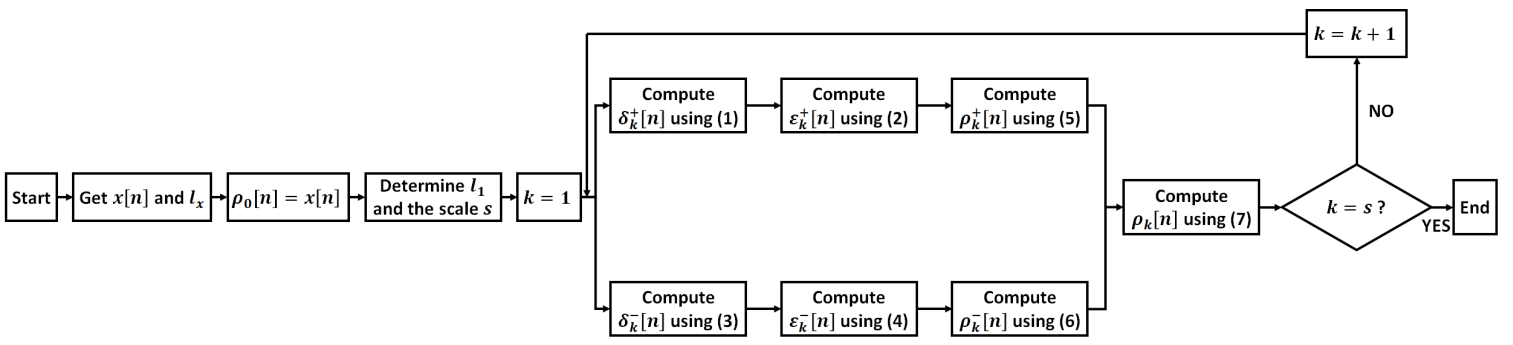

Figure 3. MSMGA flowchart

\section{MSMGA BASED ULTRA-HIGH-SPEED DIRECTIONAL PROTECTION (UHSDP)}

A lossless single-phase transmission line modeled by distributed parameters can be described mathematically by the so-called telegrapher equations:

$$
\begin{aligned}
& \frac{\partial^{2} \tilde{v}(x, t)}{\partial t^{2}}=\frac{1}{l c} \frac{\partial^{2} \tilde{v}(x, t)}{\partial x^{2}} \\
& \frac{\partial^{2} \tilde{i}(x, t)}{\partial t^{2}}=\frac{1}{l c} \frac{\partial^{2} \tilde{i}(x, t)}{\partial x^{2}}
\end{aligned}
$$

Where $l$ and $c$ are the per-unit-length inductance and capacitance respectively, $x=x_{i}-x_{j}$ represents the distance from the source, $x_{i}$ is the abscissa of the point at which the voltage is measured and $x_{j}$ is the source abscissa.

Equations (8) and (9) are also called wave equations, and their general solutions were given by D'Alembert as:

$$
\begin{aligned}
& \tilde{v}(x, t)=\tilde{v}^{+}\left(t-\frac{x}{\gamma}\right)+\tilde{v}^{-}\left(t+\frac{x}{\gamma}\right) \\
& Z \tilde{i}(x, t)=\tilde{v}^{+}\left(t-\frac{x}{\gamma}\right)-\tilde{v}^{-}\left(t+\frac{x}{\gamma}\right)
\end{aligned}
$$

Where $Z=\sqrt{l / c}$ is the surge impedance of the transmission line and $\gamma=1 / \sqrt{l c}$ is the wave velocity of propagation.

The function $\tilde{v}^{-}(t+x / \gamma)$ describes a voltage wave propagating in the negative $x$-direction, and it is called the backward voltage voltage wave. In contrast, the function $\tilde{v}^{+}(t-x / \gamma)$ describes a voltage wave propagating in the positive $x$-direction, and it is called the forward voltage wave.

As depicted in Figure 4, when a transmission line is exposed to a fault condition, the voltage $\tilde{v}(x, t)$ and the current $\tilde{i}(x, t)$ at any point of the line are subjected to two regimes [17], [18]: the steady-state regime $v(x, t)$ and $i(x, t)$ which is caused by the original sources as shown in Figure 5(a), and the fault inception regime where the superimposed quantities $\Delta v(x, t)$ and $\Delta i(x, t)$ are caused by a fictitious source exerted at the fault location $x_{F}$ and at the fault inception time $t_{F}=0$.

Therefore $\forall t \geq 0$

$$
\begin{aligned}
& \tilde{v}(x, t)=v(x, t)+\Delta v(x, t) \\
& \tilde{i}(x, t)=i(x, t)+\Delta i(x, t)
\end{aligned}
$$

We deduce from the equations above that the fault inception regime can be modeled by a network with all original sources short-circuited as shown in 5(b). We also deduce, at the fault point, that the voltage of the fictitious superimposed source and the steady-state voltage are equal in magnitude but of opposite sign. In fact, noting that $\Delta v_{F}(t)=\Delta v\left(x_{F}, t\right)$ and that we have $\tilde{v}_{F}(t)=\tilde{v}\left(x_{F}, t\right)=0$, we obtain then from equation (12) the following relation :

$$
\Delta v_{F}(t)=-v_{F}(t)
$$




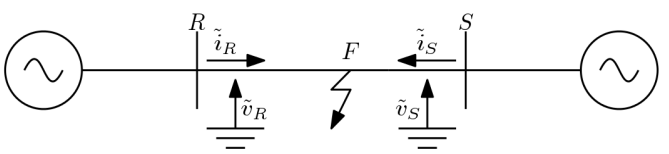

Figure 4. A transmission line subjected to a fault condition

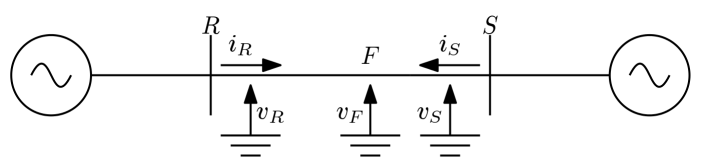

(a)

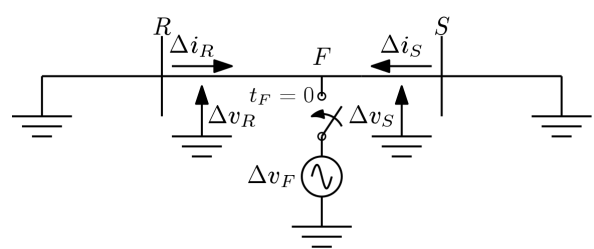

(b)

Figure 5. (a) The steady-state regime, (b) The fault inception regime

Considering that the superimposed quantities are directly related to to the fault, they should possess properties in relation to the directionality of the fault [19],[20]. In order to exhibit these properties, let's take for instance, as shown in Figure 6, a transmission system composed of two lines in which a fault is generated at point $F$ at $t_{F}=0$. According to equation (10), the forward voltage wave, $\Delta v_{F}^{+}\left(t-\left(x-x_{F}\right) / \gamma\right)$, generated by the fictitious generator, travels from the point $F$ to the discontinuity point $P$ along line 1 , the wave refracts to line 2 and the remaining reflects to line 1 . Consequently, $\Delta v_{F}^{+}\left(t-\left(x_{P}-x_{F}\right) / \gamma\right)$ is the incident wave, $\Delta v_{B}(t)$ is the reflected wave and $\Gamma_{r} \Delta v_{F}^{+}\left(t-\left(x_{P}-x_{F}\right) / \gamma\right)$ is the reflected wave, where $\Gamma_{r}=\left(Z_{2}-Z_{1}\right) /\left(Z_{2}+Z_{1}\right)$ is the coefficient of reflection, $Z_{1}$ is the surge impedance of line 1 and $Z_{2}$ is the surge impedance of line 2 .

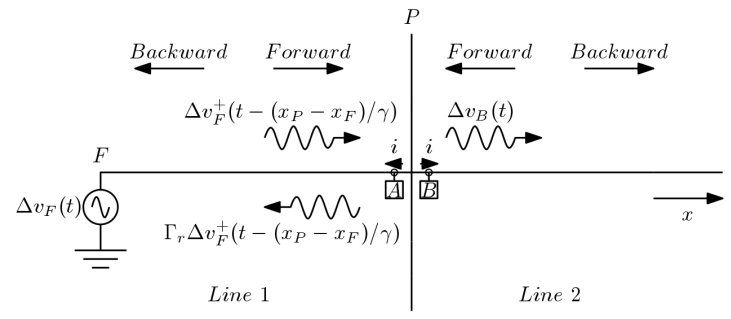

Figure 6. The fault inception regime

On Line 1, relay A measures a wave from the forward direction, namely $\Gamma_{r} \Delta v_{F}^{+}\left(t-\left(x_{P}-x_{F}\right) / \gamma\right)$, as well as one from the backward direction, that is $\Delta v_{F}^{+}\left(t-\left(x_{P}-x_{F}\right) / \gamma\right)$. Thus, we have according to relay A reference direction of the current:

$$
\begin{gathered}
\Delta v_{A}(t)=\left(1+\Gamma_{r}\right) \Delta v_{F}^{+}\left(t-\frac{x_{P}-x_{F}}{\gamma}\right) \\
-Z_{1} \Delta i_{A}(t)=\left(1-\Gamma_{r}\right) \Delta v_{F}^{+}\left(t-\frac{x_{P}-x_{F}}{\gamma}\right)
\end{gathered}
$$

Equations (14) and (15) imply the following relations:

$$
\begin{gathered}
\Delta v_{A}(t)-Z_{1} \Delta i_{A}(t) \neq 0 \\
\Delta v_{A}(t)+Z_{1} \Delta i_{A}(t)=\Gamma_{r}\left(\Delta v_{A}(t)-Z_{1} \Delta i_{A}(t)\right)
\end{gathered}
$$

We conclude that $\Delta v_{A}(t)$ and $\Delta i_{A}(t)$ are of different polarity.

On line 2 , there is no wave from the forward direction, so relay B only measures a wave from the backward direction. Thus, we obtain only, according to relay B reference direction of the current, the subsequent relation:

$$
\Delta v_{B}(t)=Z_{2} \Delta i_{B}(t)
$$


It follows from (19) that $\Delta v_{B}(t)$ and $\Delta i_{B}(t)$ have the same polarity.

This result in the following directional detection criteria:

(a) $\Delta v(x, t) \Delta i(x, t)<0 \Rightarrow$ Forward fault

(b) $\Delta v(x, t) \Delta i(x, t)>0 \Rightarrow$ Backward fault

Where $\Delta v(x, t)$ and $\Delta i(x, t)$ are the superimposed components measured at a certain discontinuity point.

Since the superimposed quantities contain information about the fault direction, as it has been shown earlier, the steady-state quantities must be removed, hence the use of the MSMGA [16]. In fact, level $s$ flat gradients $\rho_{s}^{\tilde{v}_{A}}[n], \rho_{s}^{\tilde{i}_{A}}[n], \rho_{s}^{\tilde{v}_{B}}[n]$ and $\rho_{s}^{\tilde{i}_{B}}[n]$ are extracted from the sampled signals $\tilde{v}_{A}[n], \tilde{i}_{A}[n], \tilde{v}_{B}[n]$ and $\widetilde{i}_{B}[n]$, respectively. They represent the superimposed (or transient) components, and replacing $\Delta v_{A}(t), \Delta i_{A}(t)$, $\Delta v_{B}(t)$ and $\Delta i_{B}(t)$ in (17), (18) and (19), the fault direction can be determined in practice according to the following rules:

(a) Rule 1: $\rho_{s}^{\tilde{v}}\left[k_{0}\right] \rho_{s}^{\tilde{i}}\left[k_{0}\right]<-\delta_{s} \Rightarrow$ Forward fault

(b) Rule 2: $\rho_{s}^{\tilde{v}}\left[k_{0}\right] \rho_{s}^{\tilde{i}}\left[k_{0}\right]>\delta_{s} \Rightarrow$ Backward fault

(c) Rule 3: $\left|\rho_{s}^{\tilde{v}}\left[k_{0}\right] \rho_{s}^{\tilde{i}}\left[k_{0}\right]\right|<\delta_{s} \Rightarrow$ No-fault

Where $\tilde{v}[n]$ and $\tilde{i}[n]$ are, respectively, the sampled voltage signal and the sampled current signal measured by a given relay at a certain discontinuity point. For practical reasons, the threshold $\delta_{s}>0$ should be predefined to determine the zero value of level $s$ flat gradients.

It should also be noted that $k_{0}$ is the sample index that corresponds to the local modulus maximum of the signal $\left|\rho_{s}^{\tilde{v}}[n] \rho_{s}^{\tilde{i}}[n]\right|$. It can be defined as follows:

$$
k_{0}=\arg \max _{k_{i} \leq n \leq k_{f}}\left\{\left|\rho_{s}^{\tilde{v}}[n] \rho_{s}^{\tilde{i}}[n]\right|\right\}
$$

Where $k_{i}$ is the first sample index from which $\left|\rho_{s}^{\tilde{v}}[n] \rho_{s}^{\tilde{i}}[n]\right|$ rises above $\delta_{s}$, and $k_{f}$ is the sample index after which $\left|\rho_{s}^{\tilde{v}}[n] \rho_{s}^{\tilde{i}}[n]\right|$ drops immediately under $\delta_{s}$. That is,

$$
\left|\rho_{s}^{\tilde{v}}[n] \rho_{s}^{\tilde{i}}[n]\right| \geq \delta_{s}, k_{i} \leq n \leq k_{f}
$$

\section{SIMULATION RESULTS AND DISCUSSION}

\subsection{Simulation model}

Figure 7 shows the $(440 \mathrm{kV}, 60 \mathrm{~Hz})$ transmission line system on which simulation studies had been carried out. The system is composed of two Thévenin power system equivalents and three identical transmission lines (Line 1, Line 2, Line 3) separated by two buses $R$ and $S$ modeled as short transmission lines.

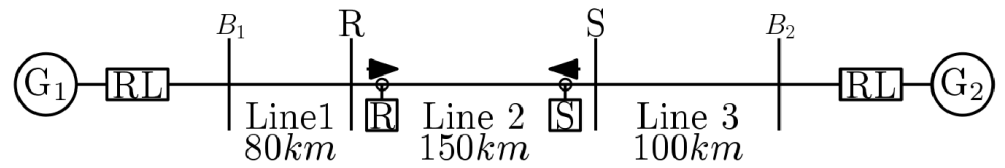

Figure 7. Simulation model

The data of the Thévenin power system equivalents are listed in the Table 1, the distributed parameters of Line 1, Line 2 and Line 3 are listed in Table 2, and The lumped parameters of the nominal- $\pi$ representation of buses $\mathrm{R}$ and $\mathrm{S}$ are given by the resistance, inductance and capacitance matrices.

Table 1. Thévenin power system equivalents data

\begin{tabular}{cccc}
\hline \multirow{2}{*}{ Generators } & \multirow{2}{*}{ Voltage $(k V)$} & \multicolumn{2}{c}{ Thévenin impedance } \\
& & Positive Seq. $(\Omega)$ & Zero Seq. $(\Omega)$ \\
\hline G1 & 462 & $19.45 \angle 84.99^{\circ}$ & $4.24 \angle 85.25^{\circ}$ \\
G2 & 418 & $20.47 \angle 84.99^{\circ}$ & $4.65 \angle 85^{\circ}$ \\
\hline
\end{tabular}


Table 2. Transmission line data

\begin{tabular}{cccc}
\hline Impedance & $\mathrm{r}(\Omega / \mathrm{km})$ & $1(\mathrm{mH} / \mathrm{km})$ & $\mathrm{c}(\mu \mathrm{F} / \mathrm{km})$ \\
\hline Positive Sequence $(\Omega)$ & 0.039 & 0.740 & 0.0157 \\
Zero Sequence $(\Omega)$ & 1.861 & 2.229 & 0.009 \\
\hline
\end{tabular}

(a) Resistance matrix $(\Omega)$ :

$$
\mathbf{R}_{\pi}=\left(\begin{array}{lll}
1.783 & 0.148 & 0.144 \\
0.148 & 1.868 & 0.148 \\
0.144 & 0.148 & 1.783
\end{array}\right)
$$

(b) Inductance matrix $(m H)$ :

$$
\mathbf{L}_{\pi}=\left(\begin{array}{lll}
9.104 & 5.118 & 4.435 \\
5.118 & 9.078 & 5.118 \\
4.435 & 5.118 & 9.104
\end{array}\right)
$$

(c) Capacitance matrix $(\mu F)$ :

$$
\mathbf{C}_{\pi}=\left(\begin{array}{lll}
0.201 & 0.044 & 0.012 \\
0.044 & 0.206 & 0.044 \\
0.012 & 0.044 & 0.201
\end{array}\right)
$$

\subsection{Internal and external fault discrimination}

The analysis given in section III for a single-phase transmission line can be extended to a three-phase transmission system using modal transformation. This transformation decomposes the voltage phase sequence $\left(\tilde{v}_{a}, \tilde{v}_{b}, \tilde{v}_{c}\right)$ and the current phase sequence $\left(\tilde{i}_{a}, \tilde{i}_{b}, \tilde{i}_{c}\right)$ into three decoupled modes, namely ground mode and aerial modes. In this scheme, the aerial mode $\alpha$, obtained from Clarke transformation, will be used:

$$
\begin{aligned}
& \tilde{v}_{\alpha}(x, t)=\frac{2}{3} \tilde{v}_{a}(x, t)-\frac{1}{3} \tilde{v}_{b}(x, t)-\frac{1}{3} \tilde{v}_{c}(x, t) \\
& \tilde{i}_{\alpha}(x, t)=\frac{2}{3} \tilde{i}_{a}(x, t)-\frac{1}{3} \tilde{i}_{b}(x, t)-\frac{1}{3} \tilde{i}_{c}(x, t)
\end{aligned}
$$

It is Line 2 which is the subject of the protection. Therefore, we desire to distinguish the faults that occur outside this line from those that occur inside it. The criteria for external and internal fault discrimination, deduced from the rules established in the previous section, are presented in Table 3.

Table 3. Rules for internal and external fault discrimination

\begin{tabular}{ccc}
\hline & \multicolumn{1}{c}{ Bus R } & Bus S \\
\hline External to R & $\rho_{s}^{\tilde{v}_{\alpha R}}\left[k_{0}\right] \rho_{s}^{\tilde{i}_{\alpha R}}\left[k_{0}\right]>\delta_{s}$ & $\rho_{s}^{\tilde{v}_{\alpha S}}\left[k_{0}\right] \rho_{s}^{\tilde{i} \alpha S}\left[k_{0}\right]<-\delta_{s}$ \\
Internal & $\rho_{s}^{\tilde{v}_{\alpha R}}\left[k_{0}\right] \rho_{s}^{\tilde{i} \alpha R}\left[k_{0}\right]<-\delta_{s}$ & $\rho_{s}^{\tilde{v}_{\alpha S}}\left[k_{0}\right] \rho_{s}^{\tilde{i} \alpha S}\left[k_{0}\right]<-\delta_{s}$ \\
External to $\mathbf{S}$ & $\rho_{s}^{\tilde{v}_{\alpha R}}\left[k_{0}\right] \rho_{s}^{\tilde{i} \alpha R}\left[k_{0}\right]<-\delta_{s}$ & $\rho_{s}^{\tilde{v}_{\alpha S}}\left[k_{0}\right] \rho_{s}^{\tilde{i}_{\alpha S}}\left[k_{0}\right]>\delta_{s}$ \\
\hline
\end{tabular}

The threshold $\delta_{s}$ is defined by taking the maximum value of level $s$ flat gradients in no-fault conditions.

\subsection{Simulation settings}

For all case studies, the voltage and current signals observed at relaying positions $\mathrm{R}$ and $\mathrm{S}$ are sampled at the rate of $1 \mathrm{MHz}$ and transformed into the $\alpha$ aerial mode using relations (21) and (22). After that, level 2 flat gradients had been extracted from the $\alpha$ aerial mode voltage and current in order to carry out the simulation studies. It should also be indicated that the threshold had been set empirically at $\delta_{2}=1400$ for level 2 flat gradients in no-fault conditions.

In the present simulation study, a time-based sliding window with a length of $1200 \mu \mathrm{s}$ and a sliding interval of $600 \mu \mathrm{s}$ is used, which is long enough to observe the fault-induced transients and short enough to avoid the effects of the reflected waves from the adjacent lines. This main window corresponds to the portions of the $\alpha$ aerial mode voltage and current signal to be processed in real-time by the MSMGA. In regards to the MSMGA, we consider $l_{1}=8$, which is equivalent to a sliding sub-window that covers a period of $8 \mu s$ and moves continuously by one sample inside the main window. 


\subsection{Case studies}

The objective behind these simulation studies is to see how the MSMGA based UHSDP relays at buses $\mathrm{R}$ and $\mathrm{S}$ responds to the different critical cases that it can face: the effect of fault inception angle near zero crossing and $90^{\circ}$ [21], the effect of single phase-to-ground fault resistance and the influence of fault location and type.

\subsubsection{An internal bolted fault}

Figure 8 shows the three-phase voltages and currents observed at measurement points $\mathrm{R}$ and $\mathrm{S}$ for a bolted phase A-to-ground fault with an inception angle close to $90^{\circ}$, at a distance of $50 \mathrm{~km}$ from bus $\mathrm{R}$. The modal voltages and currents are depicted in Figure 9 and their associated signals, $\rho_{2}^{\tilde{v}_{\alpha R}}[n] \rho_{2}^{\tilde{i}_{\alpha R}}[n]$ and $\rho_{2}^{\tilde{v}_{\alpha S}}[n] \tilde{i}_{2}^{\tilde{i}_{\alpha S}}[n]$, are depicted in Figures 10 and 11, respectively. It can be observed from Figures 10 and 11 that the transient characteristics of fault voltage and current, represented by the product of their level 2 flat gradients, are well brought out by the MSMGA, whereas the steady-state components are largely depressed (the area delimited by the red dotted lines). Furthermore, both local modulus maxima verify $\rho_{2}^{\tilde{v}_{\alpha R}}\left[k_{0}\right] \rho_{2}^{\tilde{i}_{\alpha R}}\left[k_{0}\right]<-\delta_{2}$ and $\rho_{2}^{\tilde{v}_{\alpha S}}\left[k_{0}\right] \rho_{2}^{\tilde{i}_{\alpha S}}\left[k_{0}\right]<-\delta_{2}$ (see the red points in the yellow areas of Fig.10 and Fig.11), which is consistent with the internal fault detection rule given in Table 3.
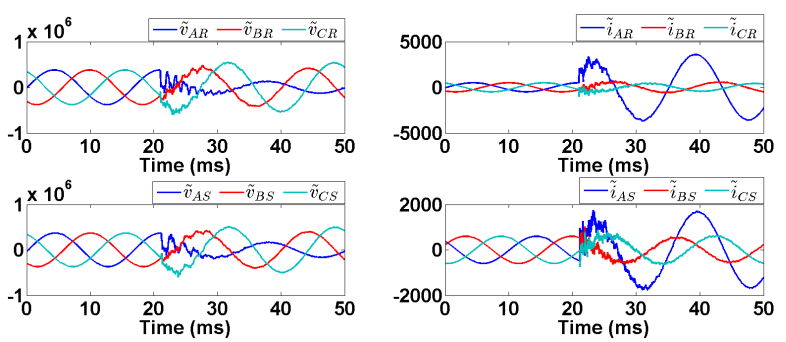

Figure 8. Three-phase voltages and currents measured at buses $\mathrm{R}$ and $\mathrm{S}$ under an internal bolted phase A-to-ground fault with a nearly $90^{\circ}$ fault inception angle at $50 \mathrm{~km}$ from bus $\mathrm{R}$

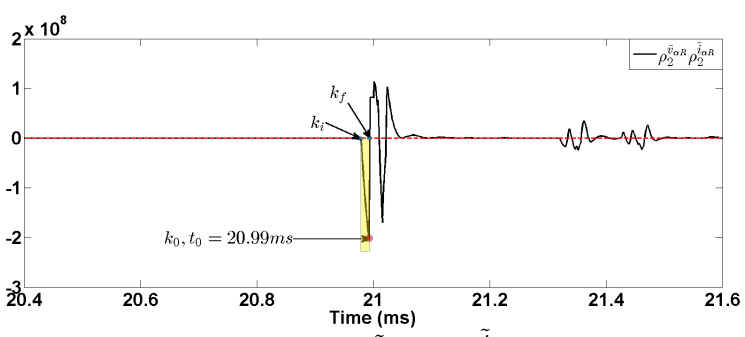

Figure 10. The signal $\rho_{2}^{\tilde{v}_{\alpha R}}[n] \rho_{2}^{\tilde{i}_{\alpha R}}[n]$ measured at bus $\mathrm{R}$ under an internal bolted phase A-to-ground fault with a nearly $90^{\circ}$ fault inception angle at $50 \mathrm{~km}$ from bus $\mathrm{R}$
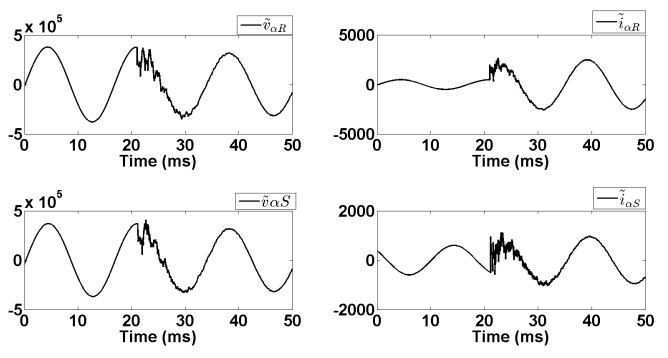

Figure 9. Modal voltages and currents measured at buses $\mathrm{R}$ and $\mathrm{S}$ under an internal

bolted phase A-to-ground fault with a nearly $90^{\circ}$ fault inception angle at $50 \mathrm{~km}$ from bus $\mathrm{R}$

\subsubsection{A high-ground resistance internal fault}

Under the same fault conditions as described in the previous case, the ground-fault resistance is increased to $200 \Omega$. The three-phase voltages and currents observed at measurement points $\mathrm{R}$ and $\mathrm{S}$ are depicted in Figure 12. The modal voltages and currents are outlined in Figure 13 and their associated signals, $\rho_{2}^{\tilde{v}_{\alpha R}}[n] \rho_{2}^{\tilde{i}_{\alpha R}}[n]$ and $\rho_{2}^{\tilde{v}_{\alpha S}}[n] \rho_{2}^{\tilde{i}_{\alpha S}}[n]$, are shown in Figures 14 and 15, respectively. The obtained results indicate that the magnitude of the local modulus maxima of the associated signals decrease with the increase of groundfault resistance. Indeed, in the previous case where the ground-resistance is near zero, the order of magnitude of the associated signals is about $10^{8}$, whereas it is about $10^{7}$ in the present case. Nevertheless, the latter order 
of magnitude is large enough to enable the detection of an internal fault (see the polarity of the red points in the yellow areas of Fig.14 and Fig.15).
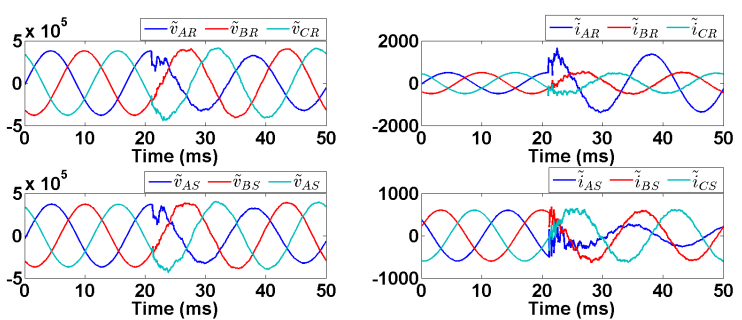

Figure 12. Three-phase voltages and currents measured at buses $\mathrm{R}$ and $\mathrm{S}$ under an internal phase A-to-ground fault with ground-resistance $200 \Omega$ at $50 \mathrm{~km}$ from bus $\mathrm{R}$

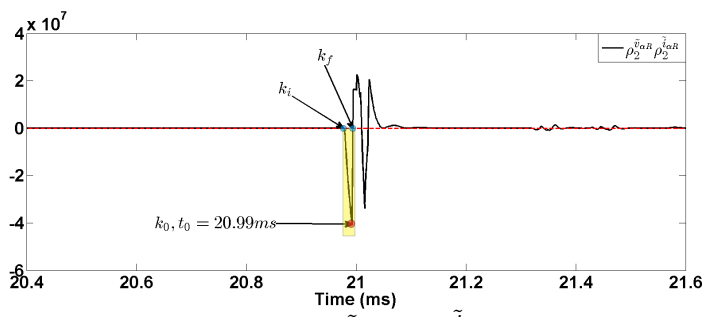

Figure 14. The signal $\rho_{2}^{\tilde{v}_{\alpha R}}[n] \rho_{2}^{\tilde{i}_{\alpha R}}[n]$ measured at bus $\mathrm{R}$ under an internal phase A-to-ground fault with ground-resistance $200 \Omega$ at $50 \mathrm{~km}$ from bus $\mathrm{R}$
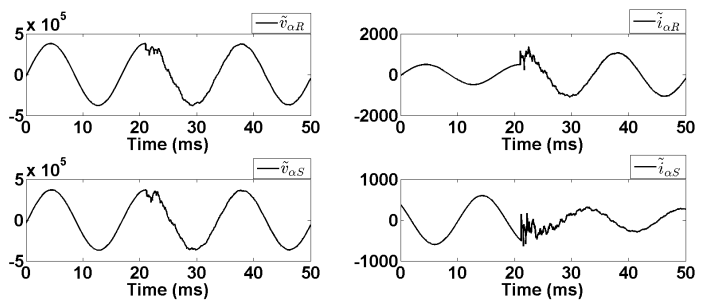

Figure 13. Modal voltages and currents measured at buses $\mathrm{R}$ and $\mathrm{S}$ under an internal phase A-to-ground fault with ground-resistance $200 \Omega$ at $50 \mathrm{~km}$ from bus

$\mathrm{R}$

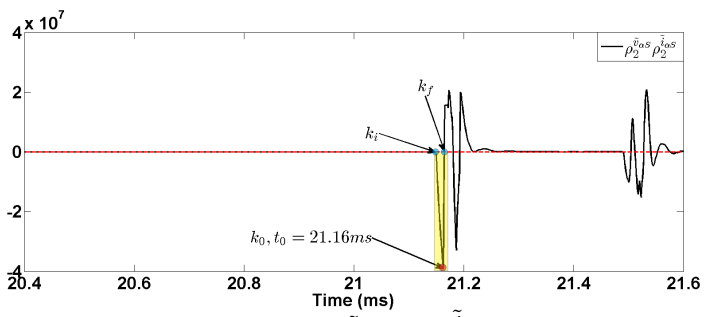

Figure 15. The signal $\rho_{2}^{\tilde{v}_{\alpha S}}[n] \rho_{2}^{\tilde{i}_{\alpha S}}[n]$ measured at bus $\mathrm{S}$ under an internal phase A-to-ground fault with ground-resistance $200 \Omega$ at $50 \mathrm{~km}$ from bus $\mathrm{R}$

\subsubsection{External faults with low inception angle}

In this case, two simulations were carried out. One concerns a fault external to bus $\mathrm{R}$ with an inception angle close to $0^{\circ}$ at a distance $25 \mathrm{~km}$ from bus $\mathrm{R}$ in Line 1 , and the other a fault external to bus $\mathrm{S}$ with an inception angle close to $0^{\circ}$ at a distance $25 \mathrm{~km}$ from bus $\mathrm{S}$ in Line 3 . Both faults occur at $16.7 \mathrm{~ms}$.

Concerning the simulation case of a fault external to bus R, Figures 16 and 17 depict the threephase voltages and current measured at points $\mathrm{R}$ and $\mathrm{S}$ and their modal components, respectively. As it can be observed, the fault-induced transients are significantly damped. Figures 18 and 19 outline the modal components associated signals, $\rho_{2}^{\tilde{v}_{\alpha R}}[n] \rho_{2}^{\tilde{i}_{\alpha R}}[n]$ and $\rho_{2}^{\tilde{v}_{\alpha S}}[n] \rho_{2}^{\tilde{i}_{\alpha S}}[n]$, respectively. From these two figures, it can be noticed that the local modulus maxima are sufficiently high to be detected. Furthermore, we have $\rho_{2}^{\tilde{v}_{\alpha R}}\left[k_{0}\right] \rho_{2}^{\tilde{i}_{\alpha R}}\left[k_{0}\right]>\delta_{2}$ and $\rho_{2}^{\tilde{v}_{\alpha S}}\left[k_{0}\right] \rho_{2}^{\tilde{i}_{\alpha S}}\left[k_{0}\right]<-\delta_{2}$ (see the red points in the yellow areas of Fig. 18 and Fig.19), which is consistent with the detection rule of a fault external to bus $\mathrm{R}$ as indicated in Table 3.
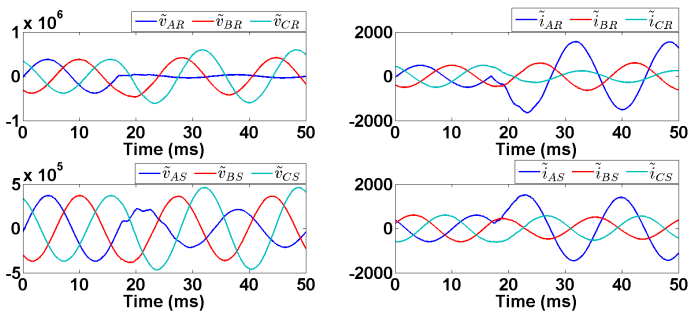

Figure 16. Three-phase voltages and currents measured at buses $\mathrm{R}$ and $\mathrm{S}$ under a phase A-to-ground fault external to bus $\mathrm{R}$ with an inception angle close to $0^{\circ}$
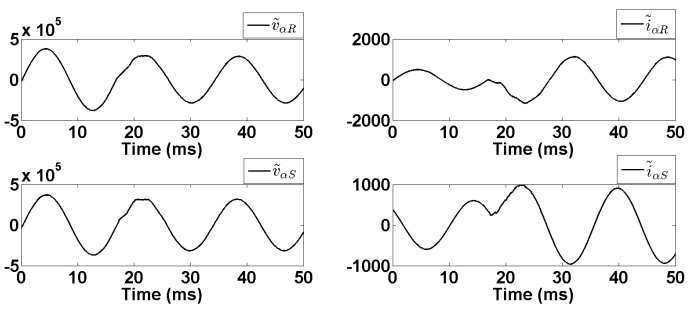

Figure 17. Modal voltages and currents measured at buses $\mathrm{R}$ and $\mathrm{S}$ under a phase A-to-ground fault external to bus $\mathrm{R}$ with an inception angle close to $0^{\circ}$ 


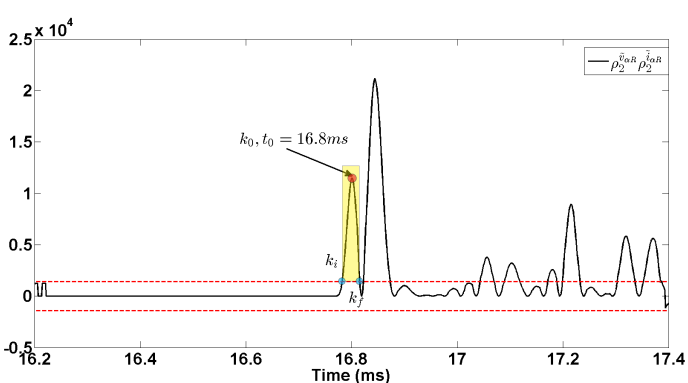

Figure 18. The signal $\rho_{2}^{\tilde{v}_{\alpha R}}[n] \rho_{2}^{\tilde{i}_{\alpha R}}[n]$ measured at bus $\mathrm{R}$ under a phase A-to-ground fault external to bus $\mathrm{R}$ with an inception angle close to $0^{\circ}$

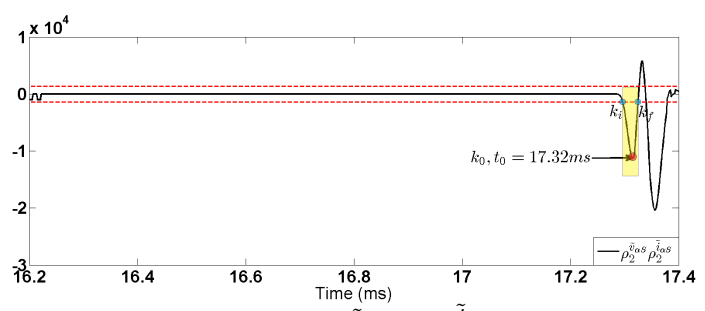

Figure 19. The signal $\rho_{2}^{\tilde{v}_{\alpha S}}[n] \rho_{2}^{\tilde{i}_{\alpha S}}[n]$ measured at bus $\mathrm{S}$ under a phase A-to-ground fault external to bus $\mathrm{R}$ with an inception angle close to $0^{\circ}$

And regarding the simulation case of a fault external to bus S, Figures 20 and 21 show the threephase voltages and currents measured at points $\mathrm{R}$ and $\mathrm{S}$ and their modal components, respectively. As it can be noticed, the fault-generated transients are significantly damped. Figures 22 and 23 outline the modal components associated signals, $\rho_{2}^{\tilde{v}_{\alpha R}}[n] \rho_{2}^{\tilde{i}_{\alpha R}}[n]$ and $\rho_{2}^{\tilde{v}_{\alpha S}}[n] \rho_{2}^{\tilde{i}_{\alpha S}}[n]$, respectively. From these two figures, it can be observed that the local modulus maxima are sufficiently high to be detected. Furthermore, we have $\rho_{2}^{\tilde{v}_{\alpha R}}\left[k_{0}\right] \rho_{2}^{\tilde{i}_{\alpha R}}\left[k_{0}\right]<-\delta_{2}$ and $\rho_{2}^{\tilde{v}_{\alpha S}}\left[k_{0}\right] \rho_{2}^{\tilde{i}_{\alpha S}}\left[k_{0}\right]>\delta_{2}$ (see the red points in the yellow areas of Fig.22 and Fig.23), which is consistent with the detection rule of a fault external to bus $\mathrm{S}$ as indicated in Table 3.
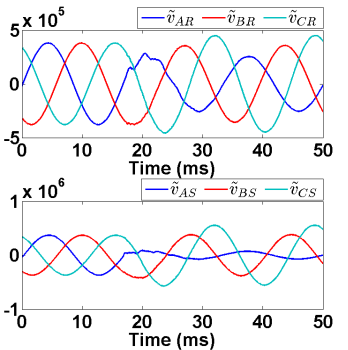

Figure 20. Three-phase voltages and currents measured at buses $\mathrm{R}$ and $\mathrm{S}$ under a phase A-to-ground fault external to bus $\mathrm{S}$ with an inception angle close to $0^{\circ}$

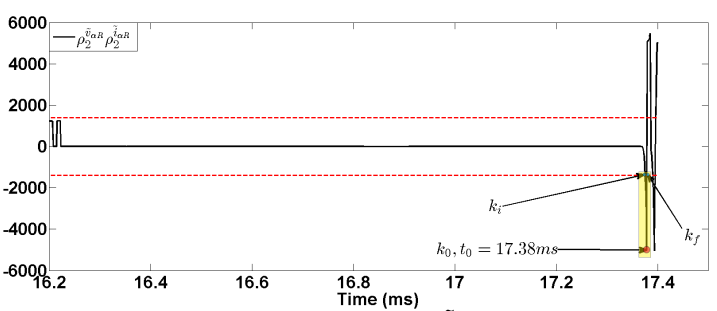

Figure 22. The signal $\rho_{2}^{\tilde{v}_{\alpha R}}[n] \rho_{2}^{\tilde{i}_{\alpha R}}[n]$ measured at bus $\mathrm{R}$ under a phase A-to-ground fault external to bus $\mathrm{S}$ with an inception angle close to $0^{\circ}$
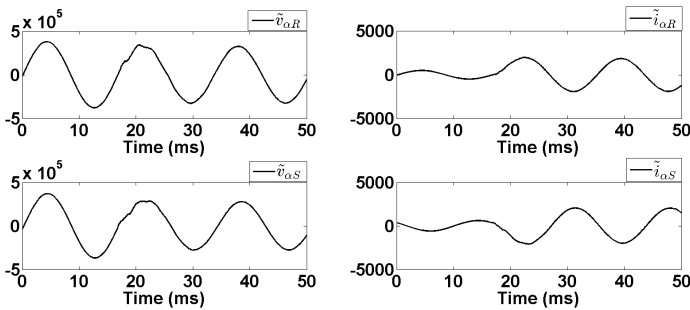

Figure 21. Modal voltages and currents measured at buses $\mathrm{R}$ and $\mathrm{S}$ under a phase A-to-ground fault external to bus $\mathrm{S}$ with an inception angle close to $0^{\circ}$

From the study of these two cases related to external faults with a fault inception angle near zero crossing, it can be deduced that the MSMGA based UHSDP is highly sensitive to signal variation. That is, the MSMGA based UHSDP is capable of detecting low amplitude fault-induced transients. 


\subsubsection{Close up bolted faults at the bus bars}

The occurrence of a fault close to the source causes the fault surge to be severely reflected and refracted between the fault point and the discontinuity points (bus bars). For the purpose of examining the MSMGA based UHSDP performance, two simulations were undertaken under the same conditions as the first case. One concerns a phase A-to-ground fault happening immediately behind the relaying position at bus $\mathrm{R}$, and the other a phase A-to-ground fault happening immediately behind the relaying position at bus $S$.

Concerning the simulation case of a fault external to bus R, Figure 24 depicts the three-phase voltages and currents measured at points $\mathrm{R}$ and $\mathrm{S}$. The modal voltages and currents are illustrated in Figure 25 and their associated signals, $\rho_{2}^{\tilde{v}_{\alpha R}}[n] \rho_{2}^{\tilde{i}_{\alpha R}}[n]$ and $\rho_{2}^{\tilde{v}_{\alpha S}}[n] \rho_{2}^{\tilde{i}_{\alpha S}}[n]$, are outlined in Figures 26 and 27), respectively. As noted in Figure 26, the yellow area corresponds to the arrival of the first front of the traveling wave, whereas the green area corresponds to the superimposition of the reflected traveling wave from the remote bus $B_{1}$ and the reflected wave at bus R. It can also be noticed from the two figures that the local modulus maxima are detected quickly and simply. Furthermore, we have $\rho_{2}^{\tilde{v}_{\alpha R}}\left[k_{0}\right] \rho_{2}^{\tilde{i}_{\alpha R}}\left[k_{0}\right]>\delta_{2}$ and $\rho_{2}^{\tilde{v}_{\alpha S}}\left[k_{0}\right] \tilde{i}_{2}^{\tilde{i}_{\alpha S}}\left[k_{0}\right]<-\delta_{2}$ (see the red points in the yellow areas of Fig.26 and Fig.27), which is consistent with the detection rule of a fault external to bus $\mathrm{R}$ as indicated in Table 3 .
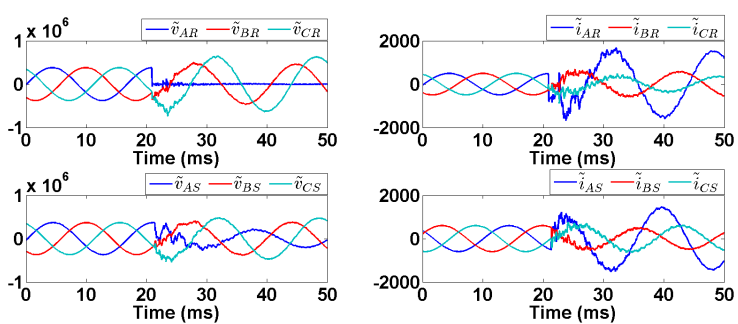

Figure 24. Three-phase voltages and currents measured at buses $\mathrm{R}$ and $\mathrm{S}$ under a phase A-to-ground fault external and close to bus R
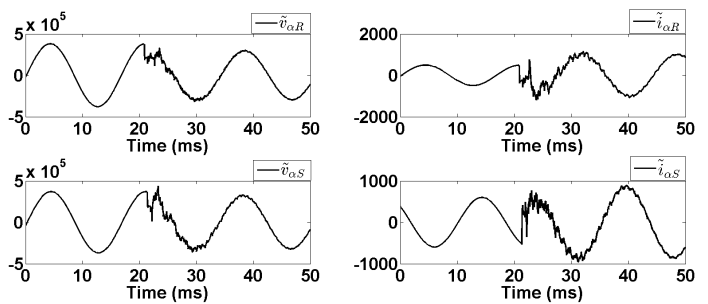

Figure 25. Modal voltages and currents measured at buses $\mathrm{R}$ and $\mathrm{S}$ under a phase A-to-ground fault external and close to bus

$\mathrm{R}$

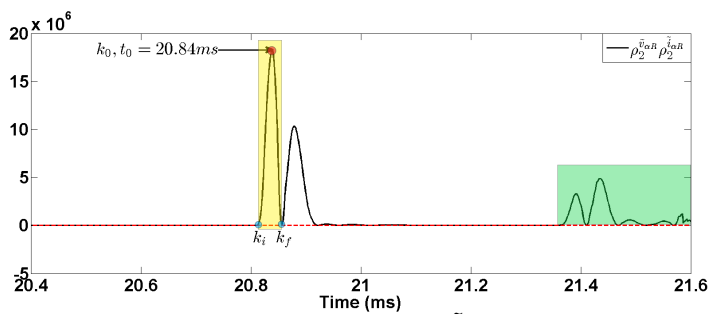

Figure 26. The signal $\rho_{2}^{\tilde{v}_{\alpha R}}[n] \rho_{2}^{\tilde{i}_{\alpha R}}[n]$ measured at bus $\mathrm{R}$ under a phase A-to-ground fault external and close close to bus $\mathrm{R}$

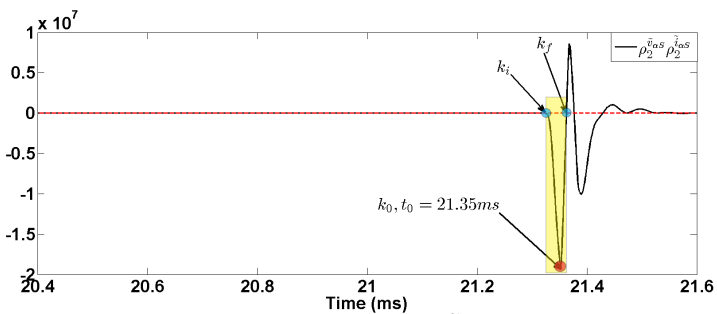

Figure 27. The signal $\rho_{2}^{\tilde{v}_{\alpha S}}[n] \rho_{2}^{\tilde{i}_{\alpha S}}[n]$ measured at bus $\mathrm{S}$ under a phase A-to-ground fault external and close to bus $\mathrm{R}$

And regarding the simulation case of a fault external to bus $\mathrm{S}$, Figure 28 shows the three-phase voltages and currents measured at points $\mathrm{R}$ and $\mathrm{S}$. The modal voltages and currents are depicted in Figure 29 and their associated signals, $\rho_{2}^{\tilde{v}_{\alpha R}}[n] \rho_{2}^{\tilde{i}_{\alpha R}}[n]$ and $\rho_{2}^{\tilde{v}_{\alpha S}}[n] \rho_{2}^{\tilde{i}_{\alpha S}}[n]$, are outlined in Figures 30 and 31, respectively. As noted in Figure 31, the yellow area corresponds to the arrival of the first front of the traveling wave, whereas the green area corresponds to the superimposition of the reflected traveling wave from the remote bus $B_{2}$ and the reflected wave at bus S. It can also be noticed from the two figures that the local modulus maxima are detected in a quick and simple way. Furthermore, we have $\rho_{2}^{\tilde{v}_{\alpha R}}\left[k_{0}\right] \rho_{2}^{\tilde{i}_{\alpha R}}\left[k_{0}\right]<-\delta_{2}$ and $\rho_{2}^{\tilde{v}_{\alpha S}}\left[k_{0}\right] \rho_{2}^{\tilde{i}_{\alpha S}}\left[k_{0}\right]>\delta_{2}$ (see the red points in the yellow areas of Fig.30 and Fig.31), which is consistent with the detection rule of a fault external to bus $\mathrm{S}$ as indicated in Table 3. 

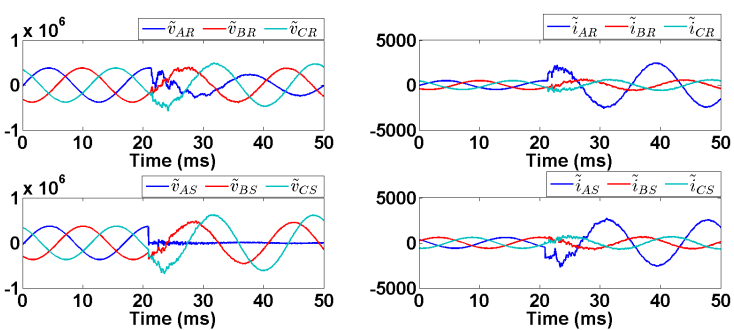

Figure 28. Three-phase voltages and currents measured at buses $\mathrm{R}$ and $\mathrm{S}$ under a phase A-to-ground fault external and close to bus $\mathrm{S}$
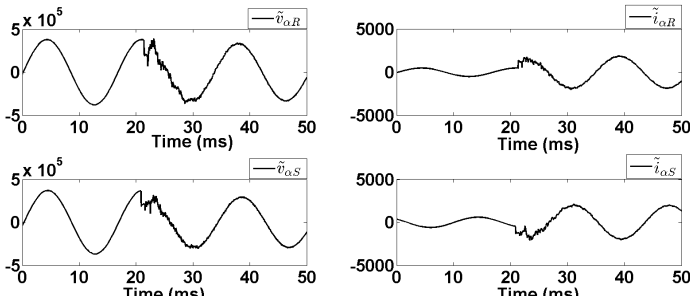

Figure 29. Modal voltages and currents measured at buses $\mathrm{R}$ and $\mathrm{S}$ under a phase A-to-ground fault external and close to bus

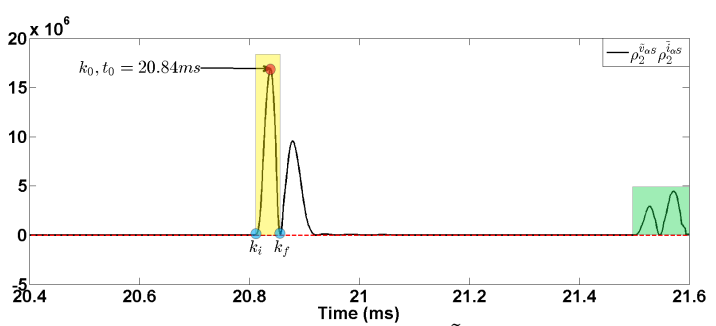

Figure 31. The signal $\rho_{2}^{\tilde{v}_{\alpha S}}[n] \rho_{2}^{\tilde{i}_{\alpha S}}[n]$ measured at bus $\mathrm{S}$ under a phase A-to-ground fault external and close to bus $\mathrm{S}$

\section{CONCLUSION}

This paper analyzes and discusses the principle and the application of the MSMGA based UHSDP for internal and external transmission line fault discrimination.

The results of the simulation studies have revealed that the proposed method overcomes all the drawbacks of the other methods mentioned in the introduction. In fact, the proposed approach provides an accurate, reliable and sensitive response to a transmission line under different critical fault conditions that have taken into consideration the modal transformations, the effect of the fault inception angle and the fault resistance as well as the influence of fault location and type.

Furthermore, the computation of the MSMGA requires only short sliding time windows as it was indicated in section 4.3. In section 4.4, it has been shown that the associated signals, $\rho_{2}^{\tilde{v}_{\alpha R}}[n] \rho_{2}^{\tilde{i}_{\alpha R}}[n]$ and $\rho_{2}^{\tilde{v}_{\alpha S}}[n] \rho_{2}^{\tilde{i}_{\alpha S}}[n]$, have a sharp shape and the polarities of their local modulus maxima are conform with the rules established in Table 3. In addition to that, it has been shown that the modulus maxima of the associated signals is able to eliminate the influence of noise. Thus, with the proposed scheme, the protection of transmission lines can be highly enhanced.

\section{REFERENCES}

[1] M. Vitins, “A fundamental concept for high speed relaying," IEEE Transactions on Power Apparatus and Systems, no. 1, pp. 163-173, 1981.

[2] A. Johns, R. Aggarwal, and Z. Bo, "Non-unit protection technique for ehv transmission systems based on fault-generated noise. part 1: signal measurement," IEE Proceedings-Generation, Transmission and Distribution, vol. 141, no. 2, pp. 133-140, 1994.

[3] Z. Bo, R. Aggarwal, A. Johns, H. Li, and Y. Song, "A new approach to phase selection using fault generated high frequency noise and neural networks," IEEE Transactions on Power Delivery, vol. 12, no. 1, pp. 106-115, 1997.

[4] E. Koley, S. K. Shukla, S. Ghosh, and D. K. Mohanta, "Protection scheme for power transmission lines based on svm and ann considering the presence of non-linear loads," IET Generation, Transmission \& Distribution, vol. 11, no. 9, pp. 2333-2341, 2017. 
[5] A. Abdullah, "Ultrafast transmission line fault detection using a dwt-based ann," IEEE Transactions on Industry Applications, vol. 54, no. 2, pp. 1182-1193, 2018.

[6] E. Khoudry, A. Belfqih, and M. N. Dazahra, "Neural network approach for fault detection in substations," in Proceedings of the 2Nd International Conference on Big Data, Cloud and Applications, ser. BDCA'17. New York, NY, USA: ACM, 2017, pp. 11:1-11:6. [Online]. Available: http://doi.acm.org/10.1145/3090354.3090365

[7] E. Khoudry, A. Belfqih, J. Boukherouaa, F. El Mariami, A. Berdai, and M. N. Dazahra, "Empirical mode decomposition and cascade feed-forward artificial neural network based intelligent fault classifier," in Smart Application and Data Analysis for Smart Cities, ser. SADASC'18, 2018.

[8] K. Amrinder, B. Yadwinder Singh, and G. Leena, "Fault detection in power transformers using random neural networks," International Journal of Electrical and Computer Engineering (IJECE), vol. 9, no. 1, pp. 78-84, 2019.

[9] A. Apostolov, "Implementation of a transient energy method for directional detection in numerical distance relays," in Transmission and Distribution Conference, 1999 IEEE, vol. 1. IEEE, 1999, pp. 382-387.

[10] T. Jinrui, Y. Xianggen, and Z. Zhe, "Modeling technology in traveling-wave fault location," Indonesian Journal of Electrical Engineering and Computer Science, vol. 11, no. 6, pp. 3333-3340, 2013.

[11] B. Rathore and A. G. Shaik, "Wavelet-alienation based transmission line protection scheme," IET Generation, Transmission \& Distribution, vol. 11, no. 4, pp. 995-1003, 2017.

[12] S. N. Ananthan, R. Padmanabhan, R. Meyur, B. Mallikarjuna, M. J. B. Reddy, and D. K. Mohanta, "Realtime fault analysis of transmission lines using wavelet multi-resolution analysis based frequency-domain approach,” IET Science, Measurement \& Technology, vol. 10, no. 7, pp. 693-703, 2016.

[13] S. Chandra Shekar, G. Ravi Kumar, and S. Lalitha, "A transient current based micro-grid connected power system protection scheme using wavelet approach," International Journal of Electrical and Computer Engineering (IJECE), vol. 9, no. 1, pp. 14-22, 2019.

[14] J.-F. Rivest, P. Soille, and S. Beucher, "Morphological gradients," Journal of Electronic Imaging, vol. 2, no. 4, pp. 326-337, 1993.

[15] L. Zhang, Y. ping Lv, and H. Yin, "A multi-resolution morphology gradient based non-communication protection scheme for transmission lines," in Electric Utility Deregulation and Restructuring and Power Technologies, 2008. DRPT 2008. Third International Conference on. IEEE, 2008, pp. 1608-1613.

[16] S. Gautam and S. M. Brahma, "Overview of mathematical morphology in power systems-a tutorial approach," in Power \& Energy Society General Meeting, 2009. PES'09. IEEE. IEEE, 2009, pp. 1-7.

[17] A. Johns, "New ultra-high-speed directional comparison technique for the protection of ehv transmission lines," in IEE proceedings C-generation, transmission and distribution, vol. 127, no. 4. IET, 1980, pp. 228-239.

[18] M. Yee and J. Esztergalyos, "Ultra high speed relay for ehv/uhv transmission lines-installation-staged fault tests and operational experience," IEEE Transactions on Power Apparatus and Systems, no. 5, pp. 1814-1825, 1978.

[19] Y. Kong, B. Zhang, and Z. Hao, "Study of ultra-high-speed protection of transmission lines using a directional comparison scheme of transient energy," IEEE Transactions on Power Delivery, vol. 30, no. 3, pp. 1317-1322, 2015.

[20] Y. Li, Y. Gong, and B. Jiang, "A novel traveling-wave-based directional protection scheme for mtdc grid with inductive de terminal," Electric Power Systems Research, vol. 157, pp. 83-92, 2018.

[21] F. Costa, B. Souza, and N. Brito, "Effects of the fault inception angle in fault-induced transients," IET generation, transmission \& distribution, vol. 6, no. 5, pp. 463-471, 2012. 


\section{BIOGRAPHIES OF AUTHORS}

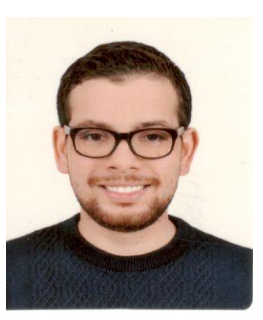

Elmahdi Khoudry is a Ph.D. student at the National Higher School of Electricity and Mechanics (University Hassan II of Casablanca- Morocco). His research activities concern power systems protection and automation as well as Smart Grids technologies.

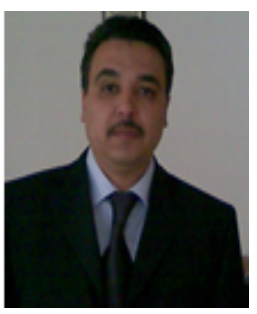

Abdelaziz Belfqih Professor at the National High School of Electricity and Mechanics (University Hassan II of Casablanca - Morocco). PhD, Engineer and holder of the University Habilitation searches (HDR). Head of the research team "Electrical Networks and Static Converters." Teacher researcher currently working on electricity network and smart grids.

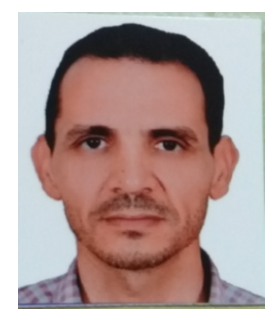

Tayeb Ouaderhman Assistant professor at Polydisciplinary faculty of Khouribga. Sultan My Slimane University. Operation research PhD. Currently working on data science and decision aid.

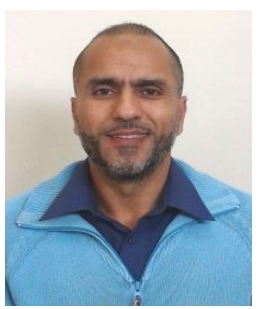

Jamal Boukherouaa Professor at the National High School of Electricity and Mechanics (University Hassan II of Casablanca - Morocco). PhD, Engineer and holder of the University Habilitation searches (HDR). Head of the research team "Electrical Networks and Static Converters." Teacher researcher currently working on high frequency static converters.

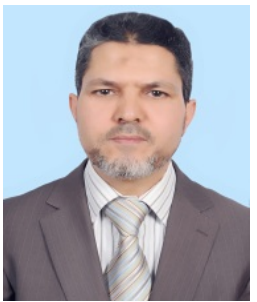

Faissal Elmariami Professor at the Superior National High School of Electricity and Mechanics (University Hassan II of Casablanca - Morocco). PhD, Engineer and holder of the University Habilitation searches (HDR). Head of the research team "Electrical Networks and Static Converters." Teacher researcher currently working on stability of the electricity network and smart grids. 